|| Print ISSN: 2589-7837 || Online ISSN: 2581-3935 ||

International Journal of Medical Science and Diagnosis Research (IJMSDR)

Available Online at www.ijmsdr.com

NLM (National Library of Medicine ID: 101738824)

Case Report

Volume 5, Issue 6; June:2021; Page No. 98-101

\title{
BLEACHING WITH 37.5\% HYDROGEN PEROXIDE-A CASE REPORT
}

Dr. Anil K Tomer ${ }^{1}$, Dr. Ayushi Khandelwal ${ }^{2}$, Dr. Hysum Mushtaq ${ }^{3}$, Dr. Lungdin Leima Cecelia ${ }^{4}$, Dr. Supriya Singh ${ }^{5}$, Dr. Shivangi Jain ${ }^{6}$, Dr. Kanika ${ }^{7}$

${ }^{1}$ Professor and Head, Department of Conservative Dentistry and Endodontics, Divya Jyoti College of Dental Sciences and Research, Modinagar, Uttar Pradesh.

${ }^{2-7}$ Postgraduate students, Departments of Conservative Dentistry and Endodontics, Divya Jyoti College of Dental Sciences and Research, Modinagar, Uttar Pradesh.

\section{Conflicts of Interest: Nil}

Corresponding author: Dr. Ayushi Khandelwal

DOI: https://doi.org/10.32553/ijmsdr.v5i6.823

\section{Abstract:}

Today's dentists have a variety of treatment options for restoring a smile with discoloured vital teeth. Treatment options include full crowns, veneers, and direct restorations, as well as minimally invasive procedures such as microabrasion, macroabrasion, and bleaching. Bleaching of teeth is the least invasive and conservative treatment option, so it is gaining popularity. The purpose of this case report is to describe an in-office vital tooth bleaching case in a young patient using POLA OFFICE + (37.5\%percent hydrogen peroxide gel). By the end of the second visit, the results were satisfactory, with no side effects.

Keywords: In office Bleaching, Pola Office +, Discoloration

\section{Introduction:}

Aesthetic dentistry is a branch of dentistry that focuses on the appearance of the dentition as determined by its arrangement, shape, and colour. The demand for aesthetic dentistry has risen steadily, and a person's smile has become an important part of their social attractiveness. Discoloration, particularly of the front teeth, causes a significant disruption in aesthetics and can lower a patient's self-esteem. Treatment options range from invasive procedures like crowns, veneers, and direct restoration placement to minimally invasive procedures like macroabrasion, microabrasion, and bleaching to simple prophylaxis. Tooth bleaching is one of the most cost-effective and conservative dental procedures for improving a person's smile.

Nowadays the society has been influenced by the picture perfect white smiles in the media. Selfawareness of discoloured teeth has risen by colour quality of television, movies, electronic and print media 1
Because of properties such as light scattering and adsorption, dentin plays a significant role in intrinsic tooth colour. Ordinarily Extrinsic stains develop in areas of the teeth that are less accessible to toothbrush cleaning and are linked to smoking, a diet high in tannin-rich foods (e.g. red wine, tea), and the use of cationic agents such as chlorhexidine or metal salts.

\section{Advantages of In-Office Bleaching:}

1) Faster bleaching procedure

2 Rrisk factor is eliminated because procedure is performed by a professional.

3) Desensitizers like potassium nitrate and fluoride help to reduce tooth sensitivity.

\section{Disadvantages of In-office bleaching :}

1) The bleaching procedure in the office is the most expensive of all the bleaching procedures.

2) The outcomes can be unpredictable and are dependent on a variety of factors. 


\section{Mechanism of tooth bleaching}

The whitening effect of $\mathrm{H} 2 \mathrm{O} 2$ is caused by the diffusion of free radicals produced by $\mathrm{H} 2 \mathrm{O} 2$ through enamel and dentin, which interact with the pigment and cause whitening. The free radicals break down the double bond between pigment molecules and change their configuration; as a result of this change in configuration, the optical properties of the tooth change, and the tooth appears to be less attractive.

\section{CASE REPORT}

A 22 year old female with favorable oral health had reported to department of conservative dentistry and endodontics with the chief complaint of discolouration of upper anterior teeth.it was the first time she requested for this cosmetic procedure, and after clinical examination, moderate yellowish bands of discolouration were observed with the report of high consumption of coffee and tea. The same patient was then scheduled for an in-office bleaching procedure.

First tooth vitality was carried out by using electronic pulp vitality tester for maxillary and mandibular anterior teeth, and all teeth found to be vital. Radiographic examination was also carried out for the same teeth to check for the presence of periapical pathologies. Additionally, the patient gave no history of tooth sensitivity. For a better accuracy, photography is used as a resource to control the shade progression as seen in the following images. After that tooth color shade was verified using a color shade guide (Vita Classical) by visual examination, and the color shade B1 was chosen as the best match with the patient's natural teeth.

Before beginning the bleaching procedure, oral prophylaxis and polishing were performed.

For this patient, Pola Office + was chosen. This material contains $37.5 \%$ hydrogen peroxide and potassium nitrate which acts as a desensitizer. After cleaning the teeth with pumice slurry and allowing them to air dry, a gingival barrier was applied and light cured for 20 seconds. Equal parts bleaching gel and activator were combined and mixed until a thick homogeneous mixture was formed, which was then applied to teeth with an applicator tip for 8 minutes. Two cycles were completed in one session. Using air water syr, the bleaching agent was removed.

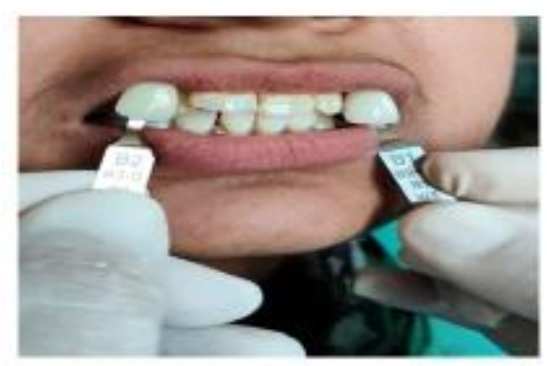

Shade selection done

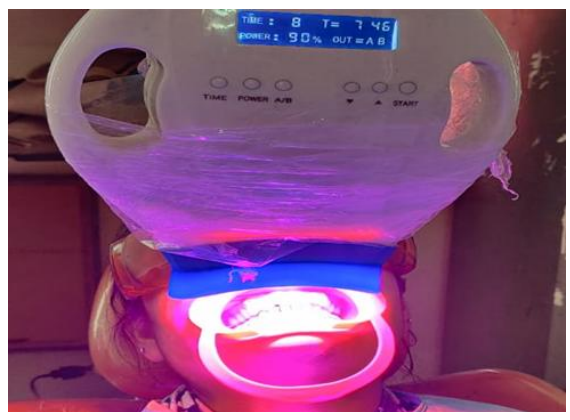

Curing of bleach

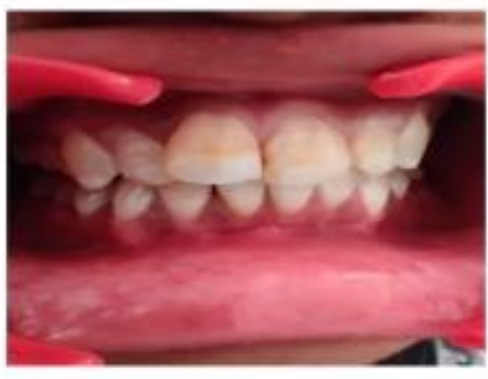

Pre operative

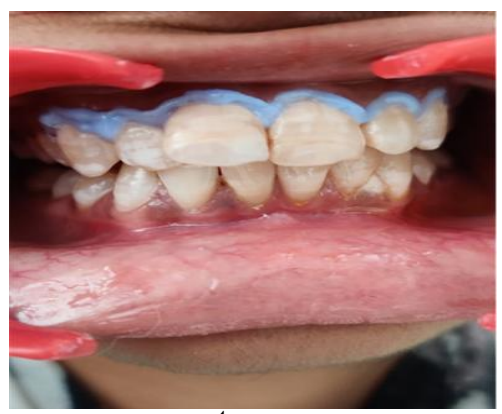

After $1^{\text {st }}$ application

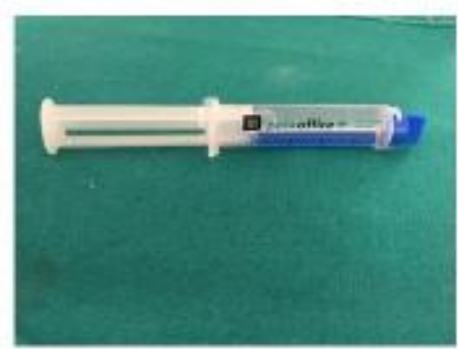

Pola office plus bleachingagent

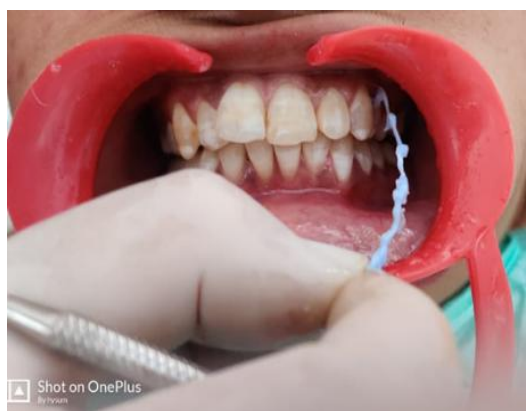

Removal of gingival barrier after $2^{\text {nd }}$ application 


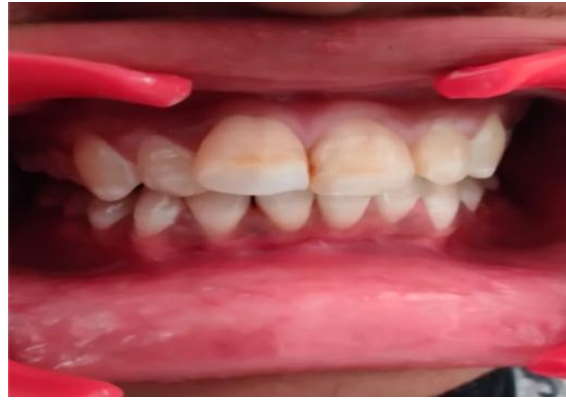

Pre-Operative

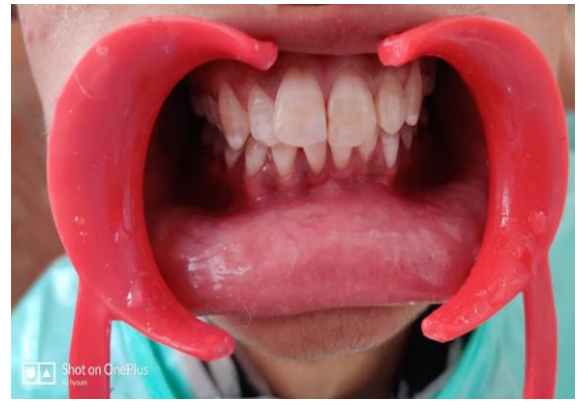

Post-Operative(After $2^{\text {nd }}$ application)

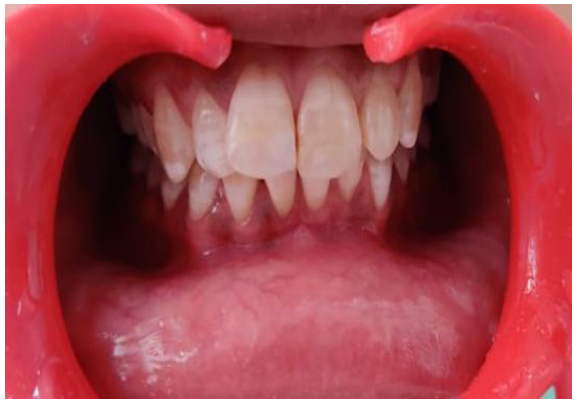

Follow up (after 3 months)

\section{Discussion}

In the field of dentistry, there are a variety of aesthetic treatment options available today, one of which is bleaching. There are several brands of bleaching agents available on the market, each with a different concentration. In this case, Pola office bleaching was used, and the results were very promising. The unique $37.5 \%$ hydrogen peroxide formula ensures fast release of the peroxide ions to begin the bleaching process.

Before beginning the bleaching procedure, a thorough clinical examination and medical history are required to determine the etiological factor for tooth discoloration as well as the degree of discoloration. Because nonvital and traumatic teeth usually appear discoloured, electric pulp testing should be performed prior to bleaching to avoid misdiagnosis and incorrect treatment. Dentists may also wish to consider prescribing NSAIDs prior to treatment since post-treatment sensitivity is unpredictable.

It is a neutral PH gel and contains desensitizers to maximize patient comfort. The dual barrel syringe system mixes as the gel is applied directly to the tooth. Pola office + is not to be used on any person having known peroxide allergies and with extremely sensitive teeth. No separate desensitizer is required .It has unique built in desensitizing properties that inhibit post-operative sensitivity. Potassium nitrate, the key painreducing ingredient, penetrates the nerve endings by blocking transmission of sensitive nerve impulses to provide a calming effect .Pulpal irritation and tooth sensitivity may be higher with the use of bleaching lights or heat application, and caution has been advised with their use. Pola office + is self - activated bleaching agent and does not require light for activation or it's optional. The underlying pulp tissue is harmed by high-concentration bleaching agents. Many in vitro studies have shown that when a bleaching agent is exposed to the tooth surface for 60 minutes, it penetrates into the pulp chamber. Hanks et al. concluded that bleaching agent penetration into pulp chamber depends on the original concentration of the bleaching agent and duration for which it has been exposed to the tooth surface, he also concluded that it took around 15 mins for bleaching agent to reach into the pulp chamber ${ }^{2}$. As molecular size and weight of peroxide, molecule is very low and has the ability to denature the protein present in dentin that's why it moves easily through dentinal tubules and reach to the pulp chamber. Anderson reported heameoxygenase 1 enzyme is protective enzyme present in endothelial cell and odontoblasts present near bleached enamel and prevents the diffusion of bleaching molecule into the pulp chamber ${ }^{1}$. Another factor responsible for the diffusion of bleaching molecule into the pulp chamber is positive pressure within the pulp chamber and osmotic pressure of the bleaching agent ${ }^{3}$.

\section{Conclusion}

In-office tooth bleaching procedure is one of the safest and quickest ways to whiten your teeth. According to newer bleaching materials that have entered the field of dentistry, in-office bleaching is safe and has no adverse effects on tooth 
structure when the bleaching agent is used in the proper concentration and the instructions are followed.

\section{References}

1. Himanshu Dixit1, Mayank Bachkaniwala2, Shahebaz khan3, Hema Yadav4,Varsha Pandit5, Jyoti Mandlik 6. In Office Teeth Whitening: Case Report: International Journal of Oral Health and Medical Research | issn 2395-7387 | SeptemberOctober 2016 | vol 3 | issue 3

2. A.R Benneti et al In vitro penetration of the bleaching agents into the pulp chamber. International endodontic journal 2004

3. Samira Esteves Afonso Camargo, MSc,* Marcia Carneiro Valera, MSc, PhD, Penetration of $38 \%$ Hydrogen Peroxide into the Pulp Chamber in Bovine and Human Teeth Submitted to Office Bleach Technique. JOE-Volume 33, Number 9, September 2007
4. 1Dr. Sadashiv G. Daokar,Dr. Aishwarya R. Mantri,Dr. Kalpana S. Patil, Dr. Kapil D. Wahane. Dr. Dimple Jethwani, Dr. Karishma Patel. In Office teeth bleaching: A case report: IJMSIR Volume - 5, Issue 5, September - 2020, Page No. : 203 - 207

5. Raghavendra Shanbhag, R Veena, Girish Nanjannawar, Jaykumar Patil, Santosh Hugar, Hemanth Vagrali. Use of Clinical Bleaching with $35 \%$ Hydrogen Peroxide in Esthetic Improvement of Fluorotic Human Incisors in vivo: The Journal of Contemporary Dental Practice, March-April 2013;14(2):208-216

6. Neha Bhutani, Bhuvan Shome Venigalla, Jaya Prakash Patil, Thakur Veerandar Singh, Sistla Venkata Jyotsna,Abhilasha Jain. Evaluation of bleaching efficacy of $37.5 \%$ hydrogen peroxide on human teeth using different modes of activations: An in vitro study: (C) 2016 Journal of Conservative Dentistry | May-Jun 2016 | Vol 19 | Issue 3 